\title{
Multicaloric effect in bi-layer multiferroic composites
}

\author{
M.M. Vopson ${ }^{1 *}$, D. Zhou ${ }^{2}$, G. Caruntu ${ }^{3}$ \\ ${ }^{1}$ University of Portsmouth, Faculty of Science, Portsmouth PO1 3QL, United Kingdom \\ ${ }^{2}$ School of Materials Science and Engineering, Dalian University of Technology, Dalian 116024, China \\ ${ }^{3}$ Department of Chemistry \& Biochemistry, Central Michigan University, Mount Pleasant, MI 48858, USA
}

\begin{abstract}
The multicaloric effect was theoretically proposed in 2012 and, despite numerous follow up studies, the effect still awaits experimental confirmation. The main limitation is the fact that the multicaloric effect is only observed at a temperature equal to the transition temperature of the magnetic and electric phases coexisting within a multiferroic (MF) (i.e. $T \approx T_{c}^{m} \approx T_{c}^{e}$ ). Such condition is hard to fulfil in single phase MFs and a solution is to develop suitable composite MF materials. Here we examine the multicaloric effect in a bi-layer laminated composite MF in order to determine the optimal design parameters for best caloric response. We show that magnetically induced multicaloric effect requires magnetic component of heat capacity smaller than that of the electric phase, while the layer thickness of the magnetic phase must be at least 5 times the thickness of the electric phase. The electrically induced multicaloric effect requires the magnetic layer to be $10 \%$ of the electric phase thickness, while its heat capacity must be larger than that of the electric phase. These selection rules are generally applicable to bulk, as well as thin film MF composites for optimal multicaloric effect.
\end{abstract}

Multiferroic (MF) materials are the subject of intense research as they are prime candidates for novel applications such as photovoltaics, electronics, opto-electronics, spintronics, energy harvesting, microwave devices, sensors and data storage, as detailed in this review article [1]. Recently, a new potential use of MFs for ultra-efficient solid-state cooling has been proposed. This application is based on the multicaloric effect, which was proposed theoretically in 2012 [2] and shortly followed by similar studies [3-12]. Caloric effects, such as heating or cooling occur in physical systems because of their thermal response to adiabatic changes of variables such as volume, strain, magnetization or electric polarization. These changes can be induced by the application or removal of a specific control parameter resulting in the well-known barocaloric [13], elastocaloric [14], magnetocaloric [15] or electrocaloric effects [16]. The potential for commercialization has stimulated research efforts, culminating with the publication of the giant magnetocaloric effect [17], the giant electrocaloric effect [18], as well as other new caloric effects $[2,19,20]$. Despite promising results, solid state cooling can only be competitive with vapour compression if new caloric materials, displaying large temperature changes in small applied external fields, are identified. The recently discovered multicaloric effect is a significant breakthrough because it combines the principles of electrocaloric, magnetocaloric and elastocaloric effects into a unified process with enhanced caloric properties [12]. The multicaloric effect is defined as the adiabatic temperature change in MF materials activated by a single electric or magnetic excitation and it is mathematically described by:

$$
\begin{aligned}
& \Delta T_{e}=-\frac{T}{C} \cdot \int_{E_{i}}^{E_{i}}\left[\frac{\alpha_{e}}{\mu_{0} \chi^{m}} \cdot\left(\frac{\partial M}{\partial T}\right)_{H, E}+\left(\frac{\partial P}{\partial T}\right)_{H, E}\right] \cdot d E \\
& \Delta T_{m}=-\frac{T}{C} \cdot \int_{H_{i}}^{H_{i}}\left[\left(\frac{\partial M}{\partial T}\right)_{H, E}+\frac{\alpha_{m}}{\varepsilon_{0} \chi^{e}} \cdot\left(\frac{\partial P}{\partial T}\right)_{H, E}\right] \cdot d H
\end{aligned}
$$

where: $\mathrm{T}$ is the operation temperature, $\mu_{0}, \varepsilon_{0}$ are the permeability and permittivity of vacuum, $\chi^{n}, \chi^{e}$ are the magnetic and electric susceptibilities, $\alpha_{e}, \alpha_{m}$ are the electrically and magnetically induced magneto-electric (ME) coupling coefficients, $M$ is the magnetization, $P$ is the polarization, $\mathrm{E}, \mathrm{H}$ are the applied electric and magnetic fields and $\mathrm{C}$ is defined as the heat capacity of the system at temperature $\mathrm{T}$ and constant $\mathrm{H}$, E fields. Although in this formalism we consider static heat capacities, in reality this is a strong approximation as the heat capacity has some non-negligible variation with the applied fields. A full derivation of relations (1) and (2) is given in $[2,12]$ and they show that for a finite adiabatic change in the applied

\footnotetext{
${ }^{*}$ Formerly known as M. Vopsaroiu.

Corresponding author: Dr Melvin Vopson: melvin.vopson@ port.ac.uk
} 
external fields $E$ or $H$, a temperature change $\Delta T$ is produced, which for a MF system is larger than the equivalent temperature change corresponding to an electrocaloric or magnetocaloric material subjected to the same applied fields. This enhanced caloric effect results from the ME coupling properties of MF $\left(\alpha_{e} /\left(\mu_{0} \cdot \chi^{m}\right) \cdot(\partial M / \partial T)\right.$ and $\left(\alpha_{m} /\left(\varepsilon_{0} \cdot \chi^{e}\right) \cdot(\partial P / \partial T)\right)$. It can be shown that an upper limit of $\Delta T$ in the multicaloric effect is twice the $\Delta T$ expected for the equivalent electrocaloric or magnetocaloric effect alone, due to the fact that $\mathrm{ME}$ susceptibility is limited by $\alpha^{2}<\mu \varepsilon$ [21]. To display large $\Delta T$, a MF needs both derivatives $\partial M / \partial T$ and $\partial P / \partial T$ maximum, which occurs when the system goes through a ferroic order phase transition. Hence, the largest multicaloric effect should take place at an operating $\mathrm{T}$ in the vicinity of the Curie transition of the magnetic and electric phases (i.e. $T \approx T_{c}^{m} \approx T_{c}{ }^{e}$ ). Unfortunately, this is the most critical limitation of the multicaloric effect, since such condition is very hard to fulfil in single phase MFs. In fact, the only known MF compound that fulfils this condition by displaying identical ferroelectric and antiferromagnetic transition temperatures $\left(T_{N}{ }^{m} \approx T_{c}{ }^{e} \approx 21 \mathrm{~K}\right)$, is $\mathrm{NdCrTiO}_{5}$ [22].

Inducing multicaloric effect in MF composites offers an elegant solution to the lack of suitable single-phase MFs. This is because in composite MFs, electric and magnetic phases coexist, but they are physically separated from each other. Hence each individual ferroic phase can be carefully selected or engineered before integrated into the MF composite complex. In our case, this would allow the fabrication of individual electrically and magnetically ordered structures with similar transition temperatures (i.e. $T_{c}^{m} \approx T_{c}^{e}$ ), fulfilling the main requirement for the occurrence of the multicaloric effect. The most promising geometry is laminated MF composite [23-26] because this is suitable for bulk as well as thin film structures [27] and such geometry offers large surface contact area necessary for solid state cooling. Moreover, it is well known that laminated MF composites usually display a strong strain-mediated ME coupling coefficient [28], even at nano-scale [29], which is another requirement for a strong multicaloric effect.

In this article, the multicaloric effect in composite MFs is examined as a possible solution to the lack of single-phase MFs with identical electric and magnetic order phase transitions. We investigate the relationship between the thickness of the constitutive layers and their specific heat capacities to the overall temperature change in order to optimize the multicaloric effect. The topic of multicaloric effect in composite MFs has not yet been discussed in the scientific community.
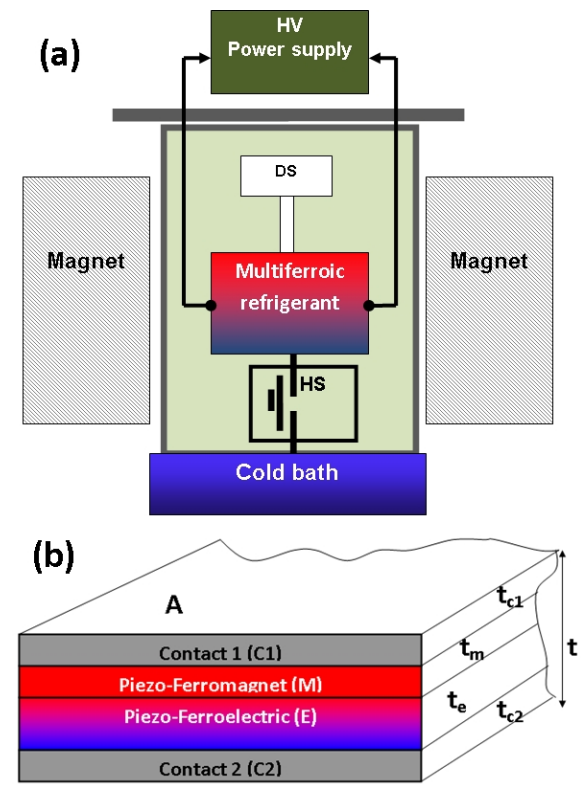

Fig. 1. (a) Schematic of the multicaloric system. HS = heat switch; DS = detector stage; HV = high voltage. $\Delta \mathrm{T}$ is measured under adiabatic demagnetization / depolarization, simultaneously. The MF refrigerant is kept adiabatically under vacuum. The HS can connect / disconnect the MF refrigerant to / from the cold bath (CB), providing the operating $\mathrm{T}$. The MF is initially magnetized / polarized while in contact with the CB. Excess heat produced due to applied $\mathrm{E} / \mathrm{H}$ is absorbed by the cold bath via the HS. The MF is then set to adiabatic conditions by opening the $\mathrm{HS}$, while the $\mathrm{E} / \mathrm{H}$ still on. This is followed by the removal of the field, which results in the adiabatic decrease of $\mathrm{T}$, as predicted by (1) and (2). (b) Proposed bi-layer laminated MF composite structure for multicaloric solid

Let us consider a cooling cycle using a multicaloric effect apparatus as shown in Fig. 1 (a) and described in its caption. The refrigerant 
is a bi-layer laminated composite MF, as shown in Fig. 1(b). The layered structure has a surface area $\mathrm{A}$ and consists of a piezo-ferromagnetic layer $(\mathrm{M})$, a piezo-ferroelectric layer $(\mathrm{E})$ and two metallic contacts / electrodes (C1 and C2). We assume the composite MF displays a nonzero strain-mediated $\mathrm{ME}$ coupling and the arbitrary magnetic and electric phases also display similar order phase transition temperatures, $T \approx T_{c}^{m} \approx T_{c}^{e}$. If $t_{m}, t_{e}, t_{c}$ is the thickness of the individual layers as shown in Fig. 1.B, and assuming identical electrodes, $t_{c l}$ $=t_{c 2}=t_{c}$, then the total thickness of the MF structure is: $t=t_{m}+t_{e}+2 t_{c}$. Equations (1) and (2) are applicable to single phase MFs, for which $\mathrm{C}$ is the heat capacity of the single-phase material. For a composite MF material of a given geometry, equations (1) and (2) must be modified to account for different heat capacities of the constitutive materials, as well as different thickness, while the bracket term under the integrals of (1) and (2) remains broadly unchanged for identical experimental conditions and materials. Hence, one would need to estimate the overall heat capacity function of the laminated MF composite in order to make predictions of the multicaloric effect in these structures. To achieve this, we apply the Matthiessen's rule for specific heat capacities of laminated structures with layers of identical surface area, taking in account contributions of individual components via a self-averaging formalism:

$\frac{1}{C}=\frac{1}{t} \sum_{i=1}^{n} \frac{t_{i}}{C_{i}}$

where $\mathrm{C}$ is the specific heat capacity of the whole layered structure, $C_{i}$ is the specific heat capacity of the $i^{\text {th }}$ layer, $t_{i}$ is the thickness of the $i^{\text {th }}$ layer and the summation is over all $n$ layers within the laminated structure. In the particular case of the bi-layer MF structure discussed in this study, see Fig. 1.B, relation (3) becomes:

$\frac{1}{C}=\frac{1}{t}\left(\frac{t_{m}}{C_{m}}+\frac{t_{e}}{C_{e}}+2 \frac{t_{c}}{C_{c}}\right)$

where $C_{m}, C_{e}$ and $C_{c}$ are the specific heat capacities of the magnetic, electric and contact layers, respectively. It is useful now to introduce the thickness ratios $\eta=\mathrm{t}_{\mathrm{m}} / \mathrm{t}_{\mathrm{e}}$ and $\xi=$ $t_{c} / t_{e}$, which combined with equation (4) results in:

$\frac{1}{C(\eta, \xi)}=\frac{1}{C_{m} C_{e} C_{c}}\left(\frac{\eta+2 \xi+1}{\eta C_{e} C_{c}+C_{c} C_{m}+2 \xi C_{e} C_{m}}\right)^{-1}$

Using (5), we can express the $\mathrm{E}$ and $\mathrm{H}$ induced multicaloric temperature changes in a bi-layer composite MF structure as:

$\Delta T_{e}=-T \cdot \frac{1}{C(\eta, \xi)} \cdot \int_{E_{i}}^{E_{i}}\left[\frac{\alpha_{e}}{\mu_{0} \chi^{m}} \cdot\left(\frac{\partial M}{\partial T}\right)_{H, E}+\left(\frac{\partial P}{\partial T}\right)_{H, E}\right] \cdot d E$
$\Delta T_{m}=-T \cdot \frac{1}{C(\eta, \xi)} \cdot \int_{H_{i}}^{H_{f}}\left[\left(\frac{\partial M}{\partial T}\right)_{H, E}+\frac{\alpha_{m}}{\varepsilon_{0} \chi^{e}} \cdot\left(\frac{\partial P}{\partial T}\right)_{H, E}\right] \cdot d H$

Hence, for the same operating temperature, the same excitation $\mathrm{E}$ and / or $\mathrm{H}$ field and the same materials with $\mathrm{C}_{\mathrm{m}}, \mathrm{C}_{\mathrm{e}}$ and $\mathrm{C}_{\mathrm{c}}$ specific heat capacities, the thickness effect of the constitutive layers to the overall multicaloric effect $\Delta \mathrm{T}_{\mathrm{e}}, \Delta \mathrm{T}_{\mathrm{m}}$ is fully described by the heat capacity function of the multicaloric composite structure, $1 / C(\eta, \xi)$. The higher the $1 / C(\eta, \xi)$, the larger the temperature change $\Delta \mathrm{T}_{\mathrm{e}}, \Delta \mathrm{T}_{\mathrm{m}}$. By studying the $1 / \mathrm{C}(\eta, \xi)$, one would be able to address the question: What is the optimal thickness of the constitutive layers within the MF composite in order to maximize the multicaloric effect for a given set of materials? We performed simulations assuming conductive $\mathrm{Cu}$ electrodes with specific heat capacity per unit volume $\mathrm{C}_{\mathrm{Cu}}=\mathrm{C}_{\mathrm{c}}=3.44 \times 10^{6}$ $\mathrm{J} / \mathrm{m}^{3} \mathrm{~K}$. For the magnetic and electric phases we used specific heat capacities ranging from $0.5 \times$ $10^{6} \mathrm{~J} / \mathrm{m}^{3} \mathrm{~K}$ to $3 \times 10^{6} \mathrm{~J} / \mathrm{m}^{3} \mathrm{~K}$. These have been selected to be smaller than the heat capacity of the $\mathrm{Cu}$ electrode, which is indeed the case in real systems as the electrode must be a very good electric conductor. Hence it is feasible to impose at all times the condition $\mathrm{C}_{\mathrm{c}}>\mathrm{C}_{\mathrm{m}}$ and $\mathrm{C}_{\mathrm{c}}>\mathrm{C}_{\mathrm{e}}$. The range of heat capacities selected are also close enough to real systems such as $\mathrm{Gd}$ and $\mathrm{Hf}_{0.2} \mathrm{Zr}_{0.8} \mathrm{O}_{2}$, both displaying excellent close to room temperature magnetocaloric [30] and electrocaloric properties [31], respectively. Gd has $\mathrm{C}_{\mathrm{Gd}}=1.85 \times 10^{6} \mathrm{~J} / \mathrm{m}^{3} \mathrm{~K}$ and Curie temperature $\mathrm{T}_{\mathrm{C}}^{\mathrm{Gd}} \approx 294 \mathrm{~K}$, while $\mathrm{T}_{\mathrm{C}}{ }^{\mathrm{HHZO}} \approx 298$ $\mathrm{K}$ and $\mathrm{C}_{\mathrm{HfZrO}}=2.13 \times 10^{6} \mathrm{~J} / \mathrm{m}^{3} \mathrm{~K}$ [31]. These materials are an example of possible constituents of a MF bi-layer composite with the structure $\mathrm{Cu} / \mathrm{Hf}_{0.2} \mathrm{Zr}_{0.8} \mathrm{O}_{2} / \mathrm{Gd} / \mathrm{Cu}$ that fulfils the requirement for the occurrence of the 
multicaloric effect at room temperature $\left(\mathrm{T}_{\mathrm{C}}{ }^{\mathrm{Gd}} \approx\right.$ $\left.\mathrm{T}_{\mathrm{C}}{ }^{\mathrm{HZZrO}} \approx 300 \mathrm{~K}\right)$. The existence of a meaningful ME coupling in such structure has not been proven so far, but this is not of concern in this study as we assume a hypothetical composite MF structure, in which a non-zero strainmediated ME coupling does occur. Fig. 2 shows the effect of the $\mathrm{Cu}$ electrode thickness ratio on the function $1 / \mathrm{C}(\eta, \xi)$ for a range of $\eta$ values when $C_{m}>C_{e}$ and when $C_{m}<C_{e}$. The graph of $1 / C(\eta, \xi)$ plotted for $C_{m}>C_{e}$, with $C_{e}$ $=0.5 \times 10^{6} \mathrm{~J} / \mathrm{m}^{3} \mathrm{~K}, \mathrm{C}_{\mathrm{m}}=3 \times 10^{6} \mathrm{~J} / \mathrm{m}^{3} \mathrm{~K}$, indicates that a thinner electrode promotes a larger $1 / C(\eta, \xi)$ function, resulting in enhanced multicaloric effect. The simulated values of the electrode thickness correspond to $10 \%, 30 \%$ and $50 \%$ of the thickness of the piezoferroelectric layer. The optimal thickness ratio for the case $C_{m}>C_{e}$ is $\xi \leq 0.1$, indicating that for a bulk sample in which piezo-ferroelectric layer is for example $t_{e}=500 \mu \mathrm{m}$, an ideal electrode must be $t_{c} \leq 50 \mu \mathrm{m}$, while in the case of a thin film composite in which the piezoferroelectric layer is $100 \mathrm{~nm}$, then a $10 \mathrm{~nm}$ electrode would be feasible. In addition, for the case $C_{m}>C_{e}$ the optimal thickness ratio $\eta=t_{m}$ $/ t_{e}$ appears to be when $\eta \leq 1$ or $t_{m} \leq t_{e}$. Hence optimal multicaloic effect for $\mathrm{C}_{\mathrm{m}}>\mathrm{C}_{\mathrm{e}}$ is achieved when $t_{m} \leq t_{e}$ and $t_{c} \leq 0.1 \cdot t_{e}$. In the same figure we plot the electrodes thickness dependence for the case $C_{e}>C_{m}$, which indicate that, again, optimal electrode thickness is $t_{c} \leq 0.1 \cdot t_{e}$, but contrary to the previous case, optimal multicaloric effect is obtained when $t_{m}$ $>5 \cdot t_{\mathrm{e}}$.

We now fix the $\mathrm{Cu}$ electrodes thickness ratio to $\xi=0.1$ and we study the effect of the heat capacity of the electric and magnetic phase on the overall heat capacity of the MF composite. Fig. 3 shows $1 / C(\eta, \xi)$ plotted as a function of $\eta$ thickness ratio when heat capacity of the magnetic phase is variable and larger than the heat capacity of the electric phase, $\mathrm{C}_{\mathrm{c}}>\mathrm{C}_{\mathrm{m}}>$ $\mathrm{C}_{\mathrm{e}}$. The heat capacity of the piezo-ferroelectric was fixed at $\mathrm{C}_{\mathrm{e}}=0.5 \times 10^{6} \mathrm{~J} / \mathrm{m}^{3} \mathrm{~K}$. Results indicate that in this case, the optimal thickness ratio $\eta=t_{m} / t_{e}$ is when $\eta \leq 1$ or $t_{m} \leq t_{e}$. When $\eta=1, \mathrm{t}_{\mathrm{m}}=\mathrm{t}_{\mathrm{e}}$ the $1 / \mathrm{C}(\eta, \xi)$ function is $25 \%$ larger for $\mathrm{C}_{\mathrm{m}}=0.75 \times 10^{6} \mathrm{~J} / \mathrm{m}^{3} \mathrm{~K}$ than for $\mathrm{C}_{\mathrm{m}}=$ $3 \times 10^{6} \mathrm{~J} / \mathrm{m}^{3} \mathrm{~K}$.

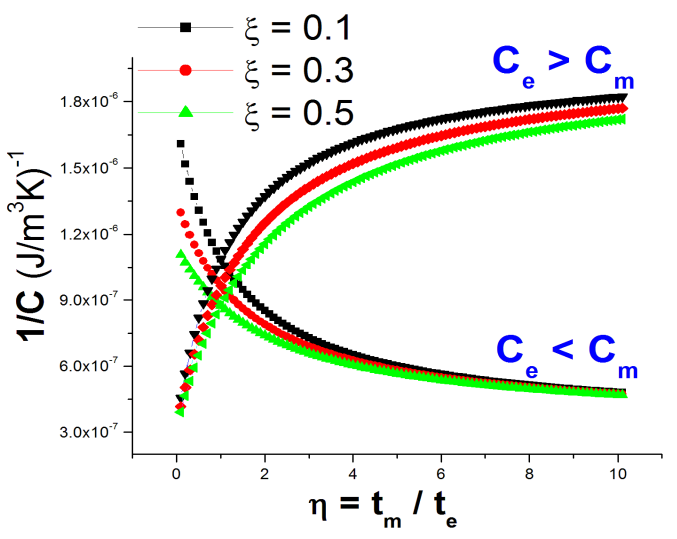

Fig. 2. Variation of the inverse heat capacity of the composite MF structure for various thickness ratio values of the contact electrodes, $\xi$, when $\mathrm{C}_{\mathrm{m}}>\mathrm{C}_{\mathrm{e}}$ and $\mathrm{C}_{\mathrm{m}}<\mathrm{C}_{\mathrm{e}}$.

However, the effect of the heat capacity of the magnetic phase is insignificant for $\eta=0.1$ or $t_{m}$ $=0.1 \cdot \mathrm{t}_{\mathrm{e}}$. We can therefore conclude that at fixed electrode thickness, $\xi=0.1$ and $\mathrm{C}_{\mathrm{c}}>\mathrm{C}_{\mathrm{m}}$ $>\mathrm{C}_{\mathrm{e}}$, the optimal geometry is $\eta=0.1$ or $\mathrm{t}_{\mathrm{m}}=$ $0.1 \cdot \mathrm{t}_{\mathrm{e}}$. In the case that a thicker magnetic layer is required, then a magnetic material with $\mathrm{C}_{\mathrm{m}}$ as close to $\mathrm{C}_{\mathrm{e}}$ as possible must be identified, while the initial condition $C_{m}>C_{e}$ is still enforced.

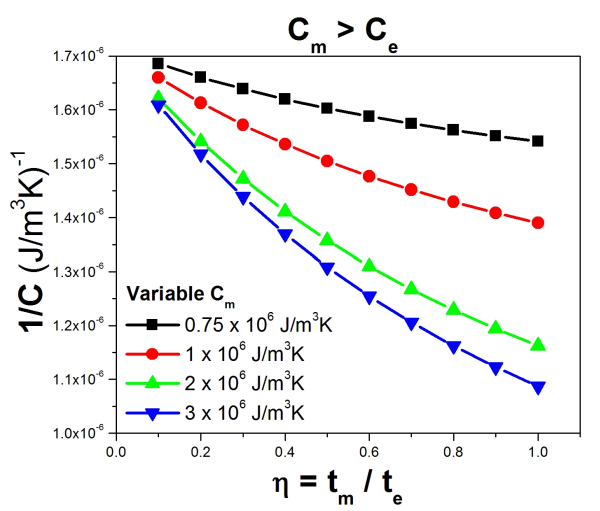

Fig. 3. Variation of the inverse heat capacity of the composite structure for various heat capacities of the magnetic material, when $\mathrm{C}_{\mathrm{m}}>\mathrm{C}_{\mathrm{e}}$ and $\xi=0.1$.

Fig. 4 shows the effect of the heat capacity of the electric phase at fixed electrode thickness, $\xi$ $=0.1$ when $\mathrm{C}_{\mathrm{c}}>\mathrm{C}_{\mathrm{e}}>\mathrm{C}_{\mathrm{m}}$. Unlike the previous case, the function $1 / \mathrm{C}(\eta, \xi)$ converges to 
almost the same value when $\eta>5$ and displays significant variations when $\eta \leq 1$ or $\mathrm{t}_{\mathrm{m}} \leq \mathrm{t}_{\mathrm{e}}$. The optimal geometry for which $1 / \mathrm{C}(\eta, \xi)$ is maximized when $\xi=0.1$ and $\mathrm{C}_{\mathrm{c}}>\mathrm{C}_{\mathrm{e}}>\mathrm{C}_{\mathrm{m}}$, is for $\eta>5$ or $t_{m}>5 \cdot t_{e}$. Should the condition $t_{m} \leq$ $t_{e}$ be necessary, then a material with $C_{m}$ value as close as possible to $\mathrm{C}_{\mathrm{e}}$, must be identified while maintaining the condition $\mathrm{C}_{\mathrm{e}}>\mathrm{C}_{\mathrm{m}}$.

Considering the two possible mechanisms of

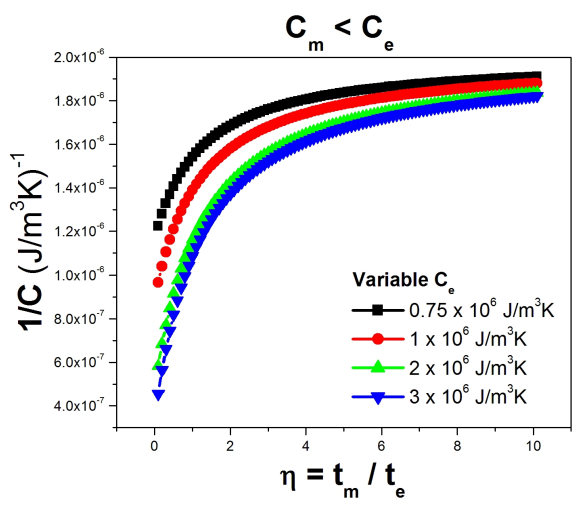

Fig. 4. Variation of the inverse heat capacity of the composite structure for various heat capacities of the piezo-ferroelectric material, when $C_{m}<C_{e}$ and $\xi=0.1$.

inducing the multicaloric effect in a $\mathrm{MF}$ composite, we summarized the results of this study in Table 1, by taking in account the fact that, if the multicaloric effect is induced electrically, then the thickness of the piezoferroelectric layer must be at least equal or larger than the thickness of the magnetic layer to ensure maximum strain-mediated $\mathrm{ME}$ coupling.

Conversely, the thickness of the magnetic layer must be at least equal or larger than the

Table 1. Recommended thickness of individual components of a laminated bi-layer MF composite for optimal multicaloric effect when $\mathrm{C}_{\mathrm{m}}>\mathrm{C}_{\mathrm{e}}$ and $\mathrm{C}_{\mathrm{m}}<\mathrm{C}_{\mathrm{e}}$ at fixed electrodes thickness, $\mathrm{t}_{\mathrm{c}}=0.1 \times \mathrm{t}_{\mathrm{e}}$, and $\mathrm{C}_{\mathrm{c}}>\mathrm{C}_{\mathrm{m}} ; \mathrm{C}_{\mathrm{c}}$ $>\mathrm{C}_{\mathrm{e}}$.

\begin{tabular}{|c|c|c|c|c|}
\hline \multirow{2}{*}{$\begin{array}{c}\text { Heat } \\
\text { capacity }\end{array}$} & \multicolumn{2}{|c|}{$\begin{array}{c}\text { H field induced } \\
\text { multicaloric effect }\end{array}$} & \multicolumn{2}{c|}{$\begin{array}{c}\text { E field induced } \\
\text { multicaloric effect }\end{array}$} \\
\cline { 2 - 5 } & $\mathrm{t}_{\mathrm{m}}>\mathrm{t}_{\mathrm{e}}$ & $\mathrm{t}_{\mathrm{m}}=\mathrm{t}_{\mathrm{e}}$ & $\mathrm{t}_{\mathrm{m}}<\mathrm{t}_{\mathrm{e}}$ & $\mathrm{t}_{\mathrm{m}}=\mathrm{t}_{\mathrm{e}}$ \\
\hline $\mathrm{C}_{\mathrm{m}}>\mathrm{C}_{\mathrm{e}}$ & - & $\mathrm{C}_{\mathrm{m}} \cong \mathrm{C}_{\mathrm{e}}$ & $\mathrm{t}_{\mathrm{m}} \cong 0.1 \times \mathrm{t}_{\mathrm{e}}$ & $\mathrm{C}_{\mathrm{m}} \cong \mathrm{C}_{\mathrm{e}}$ \\
\hline $\mathrm{C}_{\mathrm{m}}<\mathrm{C}_{\mathrm{e}}$ & $\mathrm{t}_{\mathrm{m}}>5 \times \mathrm{t}_{\mathrm{e}}$ & $\mathrm{C}_{\mathrm{m}} \cong \mathrm{C}_{\mathrm{e}}$ & - & $\mathrm{C}_{\mathrm{m}} \cong \mathrm{C}_{\mathrm{e}}$ \\
\hline
\end{tabular}

thickness of the piezo-ferroelectric layer when the multicaloric effect is magnetically driven. This study offers a clear set of design points that could be fully implemented when fabricating a laminated MF composite material for multicaloric effect studies and applications. These results are fully applicable to the fabrication of bulk laminated MFs. The same rules are also applicable to nano-thin films, if correct structures and fabrication procedures are identified to eliminate substrate thermal effects and mechanical clamping. This work is expected to stimulate further research of the multicaloric effect, especially in composite MF materials, leading to its experimental confirmation and future applications to solid state refrigeration.

\section{REFERENCES}

[1] M.M. Vopson, Critical Reviews in Solid State and Materials Sciences, 40 (4). pp. 223-250 (2015)

[2] M.M.Vopson, Solid State Communications 152, 2067-2070 (2012)

[3] H Meng, B Li, W Ren, Z Zhang, Physics Letters A, Volume 377, Issue 7, 567-571 (2013)

[4] S. Alpay, J. Mantese, S. Trolier-McKinstry, Q. Zhang, R. W. Whatmore, MRS Bulletin 39, no. 12 (2014) 1099-1111

[5] A. Planes, T. Castan, A. Saxena, Philosophical Magazine 94, no. 17 (2014) 1893-1908

[6] A. Kumar, K. L. Yadav, Journal of Applied Physics 116, no. 8 (2014): 083907

[7] I.N. Flerov, E. A. Mikhaleva, M. V. Gorev, A. V. Kartashev, Physics of the Solid State 57, no. 3 (2015): 429-441.

[8] Y. Liu, W. Jie, J. Pierre-Eymeric, I.C. Infante, J. Kreisel, X. Lou, B. Dkhil, Physical Review B 90, no. 10 (2014): 104107

[9] L.H. Yin, J. Yang, R. R. Zhang, J. M. Dai, W. H. Song, Y. P. Sun, Applied Physics Letters 104, no. 3 (2014): 032904

[10] S. Patel, A. Chauhan, R. Vaish, Applied Physics Letters 107, no. 4 (2015): 042902

[11] M.J. Krishna, A. Venimadhav, J. PhYS. D - Appl. Phys. Vol. 47, Issue 44, 445002 (2014)

[12] M.M. Vopson, J. Phys. D: Appl. Phys. 46 (2013) 345304

[13] L. Manosa, D. Gonzalez-Alonso, A. Planes, E. Bonnot, M. Barrio, J.-L. Tamarit, S. Aksoy, M. Acet, Nature Materials 9, 478 (2010)

[14] E. Bonnot, R. Romero, L. Man osa, E. Vives, A. Planes, Phys. Rev. Lett.,100, 125901 (2008)

[15] K. A. Gschneidner, V. K. Pecharsky, A. O. Tsokol, Rep. Prog. Phys. 68, 1479-1539 (2005)

[16] J.F. Scott, Electrocaloric Materials, Annu. Rev. Mater. Res. 41, 1 $12(2011)$

[17] K.V. Pecharsky, K.A. Gschneider, Giant magnetocaloric effect in $\mathrm{Gd}_{5}\left(\mathrm{Si}_{2} \mathrm{Ge}_{2}\right)$ Phys. Rev. Lett. 78, 4494 - 4497 (1997)

[18] A. S. Mischenko, Q. Zhang, J. F. Scott, R. W. Whatmore, N. D.Mathur, Science 311, 1270- 1271 (2006)

[19] T. Castan, A. Planes, A. Saxena, Phys. Rev. B 85, 144429 (2012)

[20] Z.Z. Alisultanov, L. S. Paixao, M. S. Reis, Applied Physics Letters 105, no. 23 (2014): 232406

[21] M. Fiebig, J. Phys. D: Appl. Phys. 38, (2005) R123

[22] J. Hwang, E.S. Choi, H.D. Zhou, J. lu, P. Schlottmann, Phys. Rev. B, 85, 024415 (2012)

[23] C.W. Nan, M.I. Bichurin, S. Dong, D. Viehland, G. Srinivasan, J. Appl. Phys. 103 (3), 031101 (2008)

[24] J. Zhai, Z. Xing, S. Dong, J. Li, D. Viehland, J. Am. Ceram. Soc. 91 (2) 351-358 (2008)

[25] G. Srinivasan, Annual Review of Materials Research 40, 153-178 (2010) 
[26] Y. Wang, J. Hu, Y. Lu, Ce-Wen Nan, NPG Asia Materials 2 (2) 61-68 (2010)

[27] R. Ramesh, N. A. Spaldin, Nature Materials, vol. 6, 21-29 (2007)

[28] M. Vopsaroiu, M. Stewart, T. Hegarty, A. Muniz-Piniella, N. McCartney, M. Cain, G. Srinivasan, Measurement Science \& Technology, 19, 4 (2008)

[29] G. Caruntu, A. Yourdkhani, M. Vopsaroiu, G. Srinivasan, Nanoscale, 4 (10). pp. 3218-3227 (2012)

[30] K A Gschneidner, V K Pecharsky, A O Tsokol, Rep. Prog. Phys. 68 (2005) 1479-1539

[31] M.H. Park, H.J. Kim, Y.J. Kim, T. Moon, K.D. Kim, C.S. Hwang, Nano Energy 12, 131-140 (2015) 